\title{
The twisted Daehee numbers and polynomials
}

Jin-Woo Park', Seog-Hoon Rim ${ }^{1}$ and Jongkyum Kwon ${ }^{2 *}$

${ }^{\text {*Correspondence: }}$

mathkjk26@hanmail.net

${ }^{2}$ Department of Mathematics,

Kyungpook National University,

Taegu, 702-701, Republic of Korea

Full list of author information is

available at the end of the article

\begin{abstract}
We consider the Witt-type formula for the $n$th twisted Daehee numbers and polynomials and investigate some properties of those numbers and polynomials. In particular, the nth twisted Daehee numbers are closely related to higher-order Bernoulli numbers and Bernoulli numbers of the second kind.
\end{abstract}

Keywords: the $n$th twisted Daehee numbers and polynomials; Bernoulli numbers of the second kind; higher-order Bernoulli numbers

\section{Introduction}

In this paper, we assume that $\mathbb{Z}_{p}, \mathbb{Q}_{p}$ and $\mathbb{C}_{p}$ will, respectively, denote the rings of $p$-adic integers, the fields of $p$-adic numbers and the completion of algebraic closure of $\mathbb{Q}_{p}$. The $p$-adic norm $|\cdot|_{p}$ is normalized by $|p|_{p}=1 / p$. Let $\mathrm{UD}\left[\mathbb{Z}_{p}\right]$ be the space of uniformly differentiable functions on $\mathbb{Z}_{p}$. For $f \in \mathrm{UD}\left[\mathbb{Z}_{p}\right]$, the $p$-adic invariant integral on $\mathbb{Z}_{p}$ is defined by

$$
I(f) \int_{\mathbb{Z}_{p}} f(x) d \mu_{0}(x)=\lim _{n \rightarrow \infty} \frac{1}{p^{n}} \sum_{x=0}^{p^{n}-1} f(x) \quad(\text { see }[1,2]) .
$$

Let $f_{1}$ be the translation of $f$ with $f_{1}(x)=f(x+1)$. Then, by $(1)$, we get

$$
I\left(f_{1}\right)=I(f)+f^{\prime}(0), \quad \text { where } f^{\prime}(0)=\left.\frac{d f(x)}{d x}\right|_{x=0} .
$$

As is known, the Stirling number of the first kind is defined by

$$
(x)_{n}=x(x-1) \cdots(x-n+1)=\sum_{l=0}^{n} S_{1}(n, l) x^{l},
$$

and the Stirling number of the second kind is given by the generating function to be

$$
\left(e^{t}-1\right)^{m}=m ! \sum_{l=m}^{\infty} S_{2}(l, m) \frac{t^{l}}{l !} \quad(\text { see }[3-5])
$$

For $\alpha \in \mathbb{Z}$, the Bernoulli polynomials of order $\alpha$ are defined by the generating function to be

$$
\left(\frac{t}{e^{t}-1}\right)^{\alpha} e^{x t}=\sum_{n=0}^{\infty} B_{n}^{(\alpha)}(x) \frac{t^{n}}{n !} \quad(\text { see }[3,6,7]) .
$$


When $x=0, B_{n}^{(\alpha)}=B_{n}^{(\alpha)}(0)$ are called the Bernoulli numbers of order $\alpha$.

For $n \in \mathbb{N}$, let $T_{p}$ be the $p$-adic locally constant space defined by

$$
T_{p}=\bigcup_{n \geq 1} C_{p^{n}}=\lim _{n \rightarrow \infty} C_{p^{n}}
$$

where $C_{p^{n}}=\left\{\omega \mid \omega^{p^{n}}=1\right\}$ is the cyclic group of order $p^{n}$. It is well known that the twisted Bernoulli polynomials are defined as

$$
\frac{t}{\xi e^{t}-1} e^{x t}=\sum_{n=0}^{\infty} B_{n, \xi}(x) \frac{t^{n}}{n !}, \quad \xi \in T_{p}(\text { see }[8])
$$

and the twisted Bernoulli numbers $B_{n, \xi}$ are defined as $B_{n, \xi}=B_{n, \xi}(0)$.

Recently, Kim and Kim introduced the Daehee numbers and polynomials which are given by the generating function to be

$$
\left(\frac{\log (1+t)}{t}\right)(1+t)^{x}=\sum_{n=0}^{\infty} D_{n}(x) \frac{t^{n}}{n !} \quad(\text { see }[9,10])
$$

In the special case, $x=0, D_{n}=D_{n}(0)$ are called the $n$th Daehee numbers.

In the viewpoint of generalization of the Daehee numbers and polynomials, we consider the $n$th twisted Daehee polynomials defined by the generating function to be

$$
\left(\frac{\log (1+\xi t)}{\xi t}\right)(1+\xi t)^{x}=\sum_{n=0}^{\infty} D_{n, \xi}(x) \frac{t^{n}}{n !}
$$

In the special case, $x=0, D_{n, \xi}=D_{n, \xi}(0)$ are called the $n$th twisted Daehee numbers.

In this paper, we give a $p$-adic integral representation of the $n$th twisted Daehee numbers and polynomials, which are called the Witt-type formula for the $n$th twisted Daehee numbers and polynomials. We can derive some interesting properties related to the $n$th twisted Daehee numbers and polynomials. For this idea, we are indebted to papers $[9,10]$.

\section{Witt-type formula for the $n$th twisted Daehee numbers and polynomials}

First, we consider the following integral representation associated with falling factorial sequences:

$$
\int_{\mathbb{Z}_{p}}(x)_{n} d \mu_{0}(x), \quad \text { where } n \in \mathbb{Z}_{+}=\mathbb{N} \cup\{0\} \text { (see [10]). }
$$

By (8), we get

$$
\begin{aligned}
\sum_{n=0}^{\infty} \xi^{n} \int_{\mathbb{Z}_{p}}(x)_{n} d \mu_{0}(x) \frac{t^{n}}{n !} & =\int_{\mathbb{Z}_{p}} \sum_{n=0}^{\infty} \xi^{n}\left(\begin{array}{l}
x \\
n
\end{array}\right) t^{n} d \mu_{0}(x) \\
& =\int_{\mathbb{Z}_{p}}(1+\xi t)^{x} d \mu_{0}(x),
\end{aligned}
$$

where $t \in C_{p}$ with $|t|_{p}<-\frac{1}{p-1}$. 
For $t \in C_{p}$ with $|t|_{p}<p^{-\frac{1}{p-1}}$, let us take $f(x)=(1+\xi t)^{x}$. Then, from (2), we have

$$
\int_{\mathbb{Z}_{p}}(1+\xi t)^{x} d \mu_{0}(x)=\frac{\log (1+\xi t)}{\xi t}
$$

By (9) and (10), we see that

$$
\begin{aligned}
\sum_{n=0}^{\infty} D_{n, \xi \frac{t^{n}}{n !}} & =\frac{\log (1+\xi t)}{\xi t} \\
& =\int_{\mathbb{Z}_{p}}(1+\xi t)^{x} d \mu_{0}(x) \\
& =\sum_{n=0}^{\infty} \xi^{n} \int_{\mathbb{Z}_{p}}(x)_{n} d \mu_{0}(x) \frac{t^{n}}{n !} .
\end{aligned}
$$

Therefore, by (11), we obtain the following theorem.

Theorem 1 For $n \geq 0$, we have

$$
\xi^{n} \int_{\mathbb{Z}_{p}}(x)_{n} d \mu_{0}(x)=D_{n, \xi}
$$

For $n \in \mathbb{Z}$, it is known that

$$
\left(\frac{\log (1+t)}{t}\right)^{n}(1+t)^{x-1}=\sum_{k=0}^{\infty} B_{k}^{(k-n+1)}(x) \frac{t^{k}}{k !} \quad(\text { see }[3-5])
$$

Thus, replacing $t$ by $e^{\xi t}-1$ in (12), we get

$$
D_{k, \xi}=\xi^{n} \int_{\mathbb{Z}_{p}}(x)_{k} d \mu_{0}(x)=\xi^{n} B_{k}^{(k+2)}(1) \quad(k \geq 0),
$$

where $B_{k}^{(n)}(x)$ are the Bernoulli polynomials of order $n$.

In the special case, $x=0, B_{k}^{(n)}=B_{k}^{(n)}(0)$ are called the $n$th Bernoulli numbers of order $n$.

From (11), we note that

$$
\begin{aligned}
(1+\xi t)^{x} \int_{\mathbb{Z}_{p}}(1+\xi t)^{y} d \mu_{0}(y) & =\left(\frac{\log (1+\xi t)}{\xi t}\right)(1+\xi t)^{x} \\
& =\sum_{n=0}^{\infty} D_{n, \xi}(x) \frac{t^{n}}{n !} .
\end{aligned}
$$

Thus, by (14), we get

$$
\xi^{n} \int_{\mathbb{Z}_{p}}(x+y)_{n} d \mu_{0}(y)=D_{n, \xi}(x) \quad(n \geq 0)
$$

and, from (12), we have

$$
D_{n, \xi}(x)=\xi^{n} B_{n}^{(n+2)}(x+1) .
$$

Therefore, by (15) and (16), we obtain the following theorem. 
Theorem 2 For $n \geq 0$, we have

$$
D_{n, \xi}(x)=\xi^{n} \int_{\mathbb{Z}_{p}}(x+y)_{n} d \mu_{0}(y)
$$

and

$$
D_{n, \xi}(x)=\xi^{n} B_{n}^{(n+2)}(x+1) .
$$

By Theorem 1, we easily see that

$$
D_{n, \xi}=\xi^{n} \sum_{l=0}^{n} S_{1}(n, l) B_{l}
$$

where $B_{l}$ are the ordinary Bernoulli numbers.

From Theorem 2, we have

$$
\begin{aligned}
D_{n, \xi}(x) & =\xi^{n} \int_{\mathbb{Z}_{p}}(x+y)_{n} d \mu_{0}(y) \\
& =\xi^{n} \sum_{l=0}^{n} S_{1}(n, l) B_{l}(x),
\end{aligned}
$$

where $B_{l}(x)$ are the Bernoulli polynomials defined by a generating function to be

$$
\frac{t}{e^{t}-1} e^{x t}=\sum_{n=0}^{\infty} B_{n}(x) \frac{t^{n}}{n !} .
$$

Therefore, by (17) and (18), we obtain the following corollary.

Corollary 3 For $n \geq 0$, we have

$$
D_{n, \xi}(x)=\xi^{n} \sum_{l=0}^{n} S_{1}(n, l) B_{l}(x) .
$$

In (11), we have

$$
\frac{\log (1+\xi t)}{\xi t}(1+\xi t)^{x}=\sum_{n=0}^{\infty} D_{n, \xi}(x) \frac{t^{n}}{n !} .
$$

Replacing $t$ by $e^{t}-\frac{1}{\xi}$, we put

$$
\begin{aligned}
& \sum_{n=0}^{\infty} D_{n, \xi}(x) \frac{1}{n !}\left(e^{t}-\frac{1}{\xi}\right)^{n} \\
& \quad=\frac{\log \left(1+\xi\left(e^{t}-\frac{1}{\xi}\right)\right)}{\xi\left(e^{t}-\frac{1}{\xi}\right)}\left(1+\xi\left(e^{t}-\frac{1}{\xi}\right)\right)^{x} \\
& =\frac{t}{\xi e^{t}-1}\left(\xi e^{t}\right)^{x}
\end{aligned}
$$


Therefore, we have

$$
\begin{aligned}
\sum_{n=0}^{\infty} B_{n, \xi}(x) \frac{t^{n}}{n !} & =\sum_{n=0}^{\infty} D_{n, \xi}(x) \frac{1}{n !}\left(e^{t}-\frac{1}{\xi}\right)^{n} \\
& =\sum_{n=0}^{\infty} D_{n, \xi}(x) \frac{1}{n !} \xi^{-n} n ! \sum_{m=n}^{\infty} S_{2}(m, n) \frac{t^{m}}{m !} \\
& =\sum_{m=0}^{\infty} \sum_{n=0}^{m} D_{n, \xi}(x) \xi^{-n} S_{2}(m, n),
\end{aligned}
$$

where $S_{2}(m, n)$ is the Stirling number of the second kind.

Hence,

$$
\xi^{x} B_{n, \xi}(x)=\sum_{n=0}^{m} D_{n, \xi}(x) \xi^{-n} S_{2}(m, n) .
$$

Therefore, we have

$$
B_{m, \xi}(x)=\sum_{n=0}^{m} D_{n, \xi}(x) \xi^{-n-x} S_{2}(m, n) .
$$

In particular,

$$
B_{m, \xi}=\sum_{n=0}^{m} D_{n, \xi} \xi^{-n} S_{2}(m, n) .
$$

Therefore, by (20) and (23), we obtain the following theorem.

Theorem 4 For $m \geq 0$, we have

$$
B_{m, \xi}(x)=\sum_{n=0}^{m} \xi^{-n-x} D_{n, \xi}(x) S_{2}(m, n) .
$$

In particular,

$$
B_{m, \xi}=\sum_{n=0}^{m} \xi^{-n} D_{n, \xi} S_{2}(m, n) .
$$

Remark For $m \geq 0$, by (18), we have

$$
\xi^{n} \int_{\mathbb{Z}_{p}}(x+y)^{m} d \mu_{0}(y)=\xi^{n} \sum_{n=0}^{m} D_{n}(x) S_{2}(m, n) .
$$


For $n \in \mathbb{Z}_{n \geq 0}$, the rising factorial sequence is defined by

$$
x^{(n)}=x(x+1) \cdots(x+n-1) .
$$

Let us define the $n$th twisted Daehee numbers of the second kind as follows:

$$
\widehat{D}_{n, \xi}=\xi^{n} \int_{\mathbb{Z}_{p}}(-x)_{n} d \mu_{0}(x) \quad\left(n \in \mathbb{Z}_{n \geq 0}\right) .
$$

By (26), we get

$$
x^{(n)}=(-1)^{n}(-x)_{n}=\sum_{l=0}^{n} S_{1}(n, l)(-1)^{n-l} x^{l} .
$$

From (26) and (27), we have

$$
\begin{aligned}
\widehat{D}_{n, \xi} & =\xi^{n} \int_{\mathbb{Z}_{p}}(-x)_{n} d \mu_{0}(x) \\
& =\xi^{n} \int_{\mathbb{Z}_{p}} x^{(n)}(-1)^{n} d \mu_{0}(x) \\
& =\xi^{n} \sum_{l=0}^{n} S_{1}(n, l)(-1)^{l} B_{l} .
\end{aligned}
$$

Therefore, by (28), we obtain the following theorem.

Theorem 5 For $n \geq 0$, we have

$$
\widehat{D}_{n, \xi}=\xi^{n} \sum_{l=0}^{n} S_{1}(n, l)(-1)^{l} B_{l}
$$

Let us consider the generating function of the $n$th twisted Daehee numbers of the second kind as follows:

$$
\begin{aligned}
\sum_{n=0}^{\infty} \widehat{D}_{n, \xi} \frac{t^{n}}{n !} & =\sum_{n=0}^{\infty} \xi^{n} \int_{\mathbb{Z}_{p}}(-x)_{n} d \mu_{0}(x) \frac{t^{n}}{n !} \\
& =\int_{\mathbb{Z}_{p}} \sum_{n=0}^{\infty} \xi^{n}\left(\begin{array}{c}
-x \\
n
\end{array}\right) t^{n} d \mu_{0}(x) \\
& =\int_{\mathbb{Z}_{p}}(1+\xi t)^{-x} d \mu_{0}(x) .
\end{aligned}
$$

From (2), we can derive the following equation:

$$
\int_{\mathbb{Z}_{p}}(1+\xi t)^{-x} d \mu_{0}(x)=\frac{(1+\xi t) \log (1+\xi t)}{\xi t}
$$

where $|t|_{p}<p^{-\frac{1}{p}}$ 
By (29) and (30), we get

$$
\begin{aligned}
\frac{1}{\xi t}(1+\xi t) \log (1+\xi t) & =\int_{\mathbb{Z}_{p}}(1+\xi t)^{-x} d \mu_{0}(x) \\
& =\sum_{n=0}^{\infty} \widehat{D}_{n, \xi} \frac{t^{n}}{n !} .
\end{aligned}
$$

Let us consider the $n$th twisted Daehee polynomials of the second kind as follows:

$$
\frac{(1+\xi t) \log (1+\xi t)}{\xi t} \frac{1}{(1+\xi t)^{x}}=\sum_{n=0}^{\infty} \widehat{D}_{n, \xi}(x) \frac{t^{n}}{n !}
$$

Then, by (32), we get

$$
\int_{\mathbb{Z}_{p}}(1+\xi t)^{-x-y} d \mu_{0}(y)=\sum_{n=0}^{\infty} \widehat{D}_{n, \xi}(x) \frac{t^{n}}{n !}
$$

From (33), we get

$$
\begin{aligned}
\widehat{D}_{n, \xi}(x) & =\xi^{n} \int_{\mathbb{Z}_{p}}(-x-y)_{n} d \mu_{0}(y) \quad(n \geq 0) \\
& =\xi^{n} \sum_{l=0}^{n}(-1)^{l} S_{1}(n, l) B_{l}(x) .
\end{aligned}
$$

Therefore, by (34), we obtain the following theorem.

Theorem 6 For $n \geq 0$, we have

$$
\widehat{D}_{n, \xi}(x)=\xi^{n} \int_{\mathbb{Z}_{p}}(-x-y)_{n} d \mu_{0}(y)=\xi^{n} \sum_{l=0}^{n}(-1)^{l} S_{1}(n, l) B_{l}(x)
$$

From (32) and (33), we have

$$
\frac{\log (1+\xi t)}{\xi t}(1+\xi t)^{1-x}=\sum_{n=0}^{\infty} \widehat{D}_{n, \xi}(x) \frac{t^{n}}{n !}
$$

Replacing $t$ by $e^{t}-\frac{1}{\xi}$, we get

$$
\begin{aligned}
\sum_{n=0}^{\infty} \widehat{D}_{n, \xi}(x) \frac{1}{n !}\left(e^{t}-\frac{1}{\xi}\right)^{n} & =\frac{\log \left(1+\xi\left(e^{t}-\frac{1}{\xi}\right)\right)}{\xi\left(e^{t}-\frac{1}{\xi}\right)}\left(1+\xi\left(e^{t}-\frac{1}{\xi}\right)\right)^{1-x} \\
& =\frac{t}{\xi e^{t}-1}\left(\xi e^{t}\right)^{1-x} \\
& =\xi^{1-x} \frac{t}{\xi e^{t}-1} e^{t(1-x)} \\
& =\xi^{1-x} \sum_{n=0}^{\infty} B_{n, \xi}(1-x) \frac{t^{n}}{n !}
\end{aligned}
$$


Therefore, we have

$$
\begin{aligned}
\xi^{1-x} \sum_{m=0}^{\infty} B_{m, \xi}(1-x) \frac{t^{n}}{n !} & =\sum_{n=0}^{\infty} \widehat{D}_{n, \xi}(x) \frac{\left(e^{t}-\frac{1}{\xi}\right)^{n}}{n !} \\
& =\sum_{n=0}^{\infty} \widehat{D}_{n, \xi}(x) \frac{1}{n !} \xi^{-n} n ! \sum_{m=n}^{\infty} S_{2}(m, n) \frac{t^{m}}{m !} \\
& =\sum_{m=0}^{\infty}\left(\sum_{n=0}^{m} \widehat{D}_{n, \xi}(x) \xi^{-n} S_{2}(m, n)\right) \frac{t^{m}}{m !}
\end{aligned}
$$

Hence,

$$
\xi^{1-x} B_{n, \xi}(1-x)=\sum_{n=0}^{m} \widehat{D}_{n, \xi}(x) \xi^{-n} S_{2}(m, n) .
$$

Therefore, we have

$$
B_{m, \xi}(1-x)=\sum_{n=0}^{m} \widehat{D}_{n, \xi}(x) \xi^{-n+x-1} S_{2}(m, n) .
$$

Therefore, by (37) and (38), we obtain the following theorem.

Theorem 7 For $m \geq 0$, we have

$$
B_{m, \xi}(1-x)=\sum_{n=0}^{m} \xi^{-m+x-1} \widehat{D}_{n, \xi}(x) S_{2}(m, n) .
$$

From Theorem 1 and (26), we have

$$
\begin{aligned}
(-1)^{n} \frac{D_{n, \xi}}{n !} & =(-1)^{n} \xi^{n} \int_{\mathbb{Z}_{p}}\left(\begin{array}{l}
x \\
n
\end{array}\right) d \mu_{0}(x) \\
& =\xi^{n} \int_{\mathbb{Z}_{p}}\left(\begin{array}{c}
-x+n-1 \\
n
\end{array}\right) d \mu_{0}(x) \\
& =\xi^{n} \sum_{m=0}^{n}\left(\begin{array}{c}
n-1 \\
n-m
\end{array}\right) \int_{\mathbb{Z}_{p}}\left(\begin{array}{l}
-x \\
m
\end{array}\right) d \mu_{0}(x) \\
& =\sum_{m=0}^{n}\left(\begin{array}{c}
n-1 \\
n-m
\end{array}\right) \xi^{n-m} \frac{\widehat{D}_{m, \xi}}{m !} \\
& =\sum_{m=1}^{n}\left(\begin{array}{c}
n-1 \\
m-1
\end{array}\right) \xi^{n-m} \frac{\widehat{D}_{m, \xi}}{m !}
\end{aligned}
$$

and

$$
\begin{aligned}
(-1)^{n} \frac{\widehat{D}_{n, \xi}}{n !} & =(-1)^{n} \xi^{n} \int_{\mathbb{Z}_{p}}\left(\begin{array}{c}
-x \\
n
\end{array}\right) d \mu_{0}(x) \\
& =\xi^{n} \int_{\mathbb{Z}_{p}}\left(\begin{array}{c}
x+n-1 \\
n
\end{array}\right) d \mu_{0}(x)
\end{aligned}
$$




$$
\begin{aligned}
& =\xi^{n} \sum_{m=0}^{n}\left(\begin{array}{c}
n-1 \\
n-m
\end{array}\right) \int_{0}^{1}\left(\begin{array}{c}
x \\
m
\end{array}\right) d \mu_{0}(x) \\
& =\sum_{m=0}^{n}\left(\begin{array}{c}
n-1 \\
m-1
\end{array}\right) \xi^{n-m} \frac{D_{m, \xi}}{m !} \\
& =\sum_{m=1}^{n}\left(\begin{array}{c}
n-1 \\
m-1
\end{array}\right) \xi^{n-m} \frac{D_{m, \xi}}{m !}
\end{aligned}
$$

Therefore, by (40) and (41), we obtain the following theorem.

Theorem 8 For $n \in \mathbb{N}$, we have

$$
(-1)^{n} \frac{D_{n, \xi}}{n !}=\sum_{m=1}^{n}\left(\begin{array}{c}
n-1 \\
m-1
\end{array}\right) \xi^{n-m} \frac{\widehat{D}_{m, \xi}}{m !}
$$

and

$$
(-1)^{n} \frac{\widehat{D}_{n, \xi}}{n !}=\sum_{m=1}^{n}\left(\begin{array}{c}
n-1 \\
m-1
\end{array}\right) \xi^{n-m} \frac{D_{m, \xi}}{m !} .
$$

\section{Competing interests}

The authors declare that they have no competing interests.

\section{Authors' contributions}

The authors contributed equally to the manuscript and typed, read, and approved the final manuscript.

\section{Author details}

'Department of Mathematics Education, Kyungpook National University, Taegu, 702-701, Republic of Korea.

${ }^{2}$ Department of Mathematics, Kyungpook National University, Taegu, 702-701, Republic of Korea.

\section{Acknowledgements}

The authors are grateful for the valuable comments and suggestions of the referees.

Received: 30 October 2013 Accepted: 6 December 2013 Published: 02 Jan 2014

\section{References}

1. Kim, T, Kim, DS, Mansour, T, Rim, SH, Schork, M: Umbral calculus and Sheffer sequence of polynomials. J. Math. Phys. 54, 083504 (2013). doi:10.1063/1.4817853

2. Kim, T: An invariant $p$-adic integral associated with Daehee numbers. Integral Transforms Spec. Funct. 13(1), 65-69 (2002)

3. Bayad, A: Special values of Lerch zeta function and their Fourier expansions. Adv. Stud. Contemp. Math. 21(1), 1-4 (2011)

4. Carlitz, L: A note on Bernoulli and Euler polynomials of the second kind. Scr. Math. 25, 323-330 (1961)

5. Comtet, L: Advanced Combinatorics. Reidel, Dordrecht (1974)

6. Araci, S, Acikgoz, M: A note on the Frobenius-Euler numbers and polynomials associated with Bernstein polynomials. Adv. Stud. Contemp. Math. 22(3), 399-406 (2012)

7. Kurt, V: Some relation between the Bernstein polynomials and second kind Bernoulli polynomials. Adv. Stud. Contemp. Math. 23(1), 43-48 (2013)

8. Kim, T: An analogue of Bernoulli numbers and their congruences. Rep. Fac. Sci. Eng. Saga Univ., Math. 22(2), 21-26 (1994)

9. Kim, DS, Kim, T, Lee, SH, Seo, JJ: A note on the twisted $\lambda$-Daehee polynomials. Appl. Math. Sci. 7 (2013)

10. Kim, DS, Kim, T: Daehee numbers and polynomials. Appl. Math. Sci. 7(120), 5969-5976 (2013)

10.1186/1687-1847-2014-1

Cite this article as: Park et al.: The twisted Daehee numbers and polynomials. Advances in Difference Equations 2014, 2014:1 